\title{
Beyond dreams: do sleep-related movements contribute to brain development?
}

\author{
Mark S. Blumberg* \\ Department of Psychology and Delta Center, The University of lowa, lowa City, IA, USA
}

\section{Edited by:}

Milton Kramer, University of

Illinois-Chicago, USA

\section{Reviewed by}

James F. Pagel, The American Academy

of Family Physicians, USA

Milton Kramer, University of

Illinois-Chicago, USA

*Correspondence:

Mark S. Blumberg, Department of Psychology, University of lowa,

E11 Seashore Hall, lowa City, IA 52242,

USA.

e-mail: mark-blumberg@uiowa.edu
Conventional wisdom has long held that the twitches of sleeping infants and adults are byproducts of a dreaming brain. With the discovery of active (or REM) sleep in the 1950s and the recognition soon thereafter that active sleep is characterized by inhibition of motor outflow, researchers elaborated on conventional wisdom and concluded that sleep-related twitches are epiphenomena that result from incomplete blockade of dream-related cortical activity. This view persists despite the fact that twitching is unaffected in infants and adults when the cortex is disconnected from the brainstem. In 1966, Roffwarg and colleagues introduced the ontogenetic hypothesis, which addressed the preponderance of active sleep in early infancy. This hypothesis posited that the brainstem mechanisms that produce active sleep provide direct ascending stimulation to the forebrain and descending stimulation to the musculature, thereby promoting brain and neuromuscular development. However, this hypothesis and the subsequent work that tested it did not directly address the developmental significance of twitching or sensory feedback as a contributor to activity-dependent development. Here I review recent findings that have inspired an elaboration of the ontogenetic hypothesis. Specifically, in addition to direct brainstem activation of cortex during active sleep, sensory feedback arising from limb twitches produces discrete and substantial activation of somatosensory cortex and, beyond that, of hippocampus . Delineating how twitching during active sleep contributes to the establishment, refinement, and maintenance of neural circuits may aid our understanding of the early developmental events that make sensorimotor integration possible. In addition, twitches may prove to be sensitive and powerful tools for assessing somatosensory function in humans across the lifespan as well as functional recovery in individuals with injuries or conditions that affect sensorimotor function.

Keywords: REM sleep, spontaneous activity, myoclonic twitching, sensorimotor, cortex, hippocampus, proprioception, REM behavior disorder
The theory that sleep plays a functional role in learning and memory continues to attract attention and generate controversy (Smith, 1995; Siegel, 2001; Stickgold, 2005; Korman et al., 2007). Although most attempts to test this theory have focused on adults, investigators have also explored the possibility that sleep contributes to such developmental phenomena as language learning in human infants (Hupbach et al., 2009), visual cortical plasticity in juvenile rats (Oksenberg et al., 1996; Frank et al., 2001; Shaffery et al., 2002; Aton et al., 2009), and song learning in juvenile birds (Margoliash, 2005). The authors of a recent report demonstrating classical conditioning in sleeping human infants suggested that the capacity to learn while asleep, perhaps unique to infants, reflects the enhanced plasticity of the brain early in development (Fifer et al., 2010).

In contrast with this intense and growing interest in sleep and its effects on cognitive function, it is noteworthy that one of the most striking behavioral manifestations of sleep-the twitchy, jerky movements of the limbs that characterize rapid eye movement or REM sleep (hereafter referred to as active sleep) - is largely ignored. To be sure, there are many striking features of sleep: bizarre dreams, complete loss of muscle tone, and rapid eye movements; but of these, only twitching is so readily apparent even to a casual observer. As will be explained in this review, although researchers have long thought that they grasp the significance of these limb twitches, work over the last decade has shown otherwise.
When we see a burst of jerky limb movements - known to scientists and clinicians as myoclonic twitching - many of us think we are watching the outward manifestation of a dream. George Romanes, the early comparative psychologist and protégé of Charles Darwin, endorsed this conventional wisdom when he wrote that "ferrets dream, as I have frequently seen them when fast asleep moving their noses and twitching their claws as if in pursuit of rabbits" (Romanes, 1883/1977). More recently, Cesar Millan, known to his fans as the Dog Whisperer, expressed a similar sentiment about the meaning of movements during sleep: "I don't know what dogs dream, but they are definitely doing something really fun" (Millan, 2009) (see Movie S1 in Supplementary Material).

As conspicuous as twitching is in adults, it is much more conspicuous in infants. In infant rats during active sleep, for example, bursts of skeletal muscle activity give rise to twitches of the head, limbs, and tail (see Figure 1 and Movie S2 in Supplementary Material). These bursts of twitching can be so vigorous and intense that they can sometimes be mistaken for a seizure (Holtzman et al., 1981). I once observed an infant rat flip itself over during a bout of twitching - from a supine to prone position - while remaining asleep. Twitching is also more conspicuous in newborns because they are asleep approximately two-thirds of the time, with active sleep accounting for $50 \%$ or more of the time asleep: for example, 


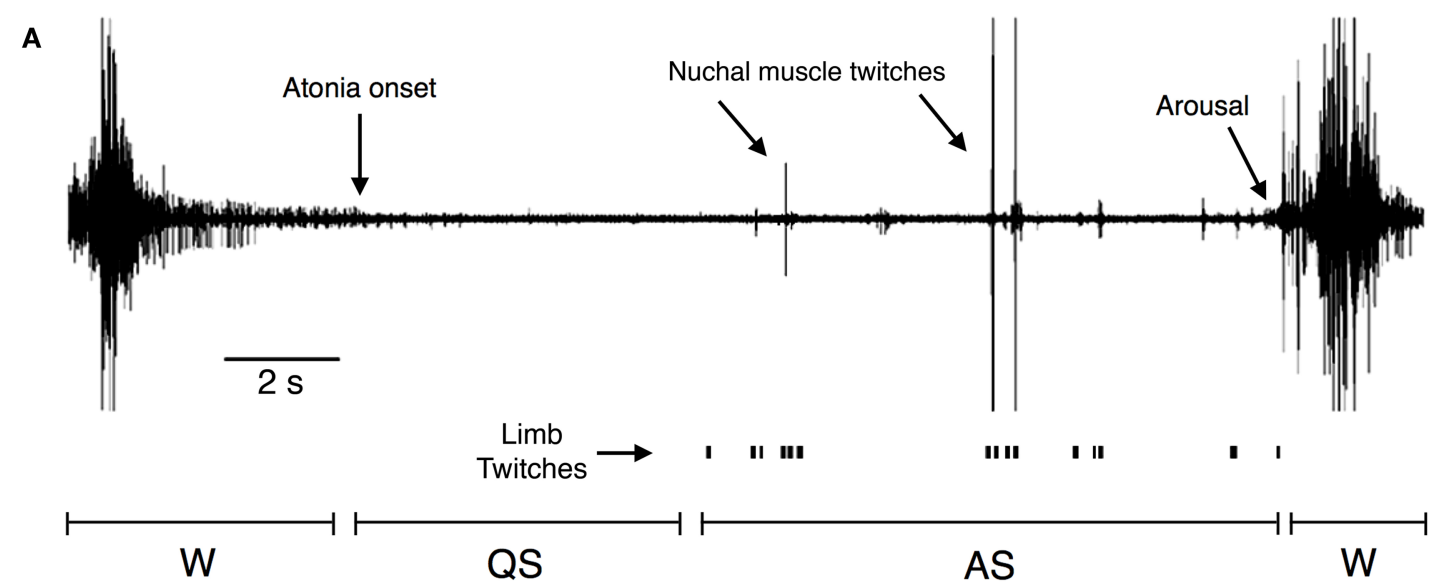

B

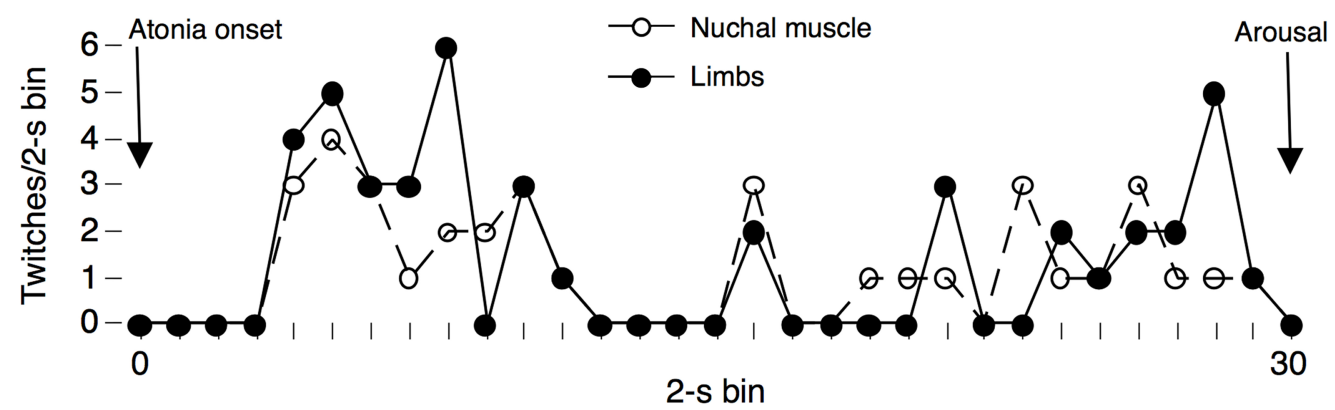

FIGURE 1 | (A) Representative cycle of high nuchal muscle tone (indicative of wakefulness) and atonia (indicative of sleep) in a P8 rat. Nuchal muscle twitches against a background of atonia are indicated, as are instances of visually scored limb twitches. This sleep-wake cycle has been divided into periods of wakefulness (W), quiet sleep (OS), and active sleep (AS). (B) Representative data from a single period of atonia in a P8 rat showing the number of twitches measured during successive 2-s bins. Electrographically scored nuchal muscle twitches (open circles) and visually scored limb twitches (filled circles) are shown separately and indicate temporally confined bursts of twitching during active sleep. Adapted from Seelke and Blumberg (2005). human newborns are in active sleep for $8 \mathrm{~h}$ each day (Roffwarg et al., 1966), and infant rats exhibit similarly high rates of sleep throughout the early postnatal period (Gramsbergen et al., 1970; Jouvet-Mounier et al., 1970; Seelke and Blumberg, 2008). Thus, in terms of intensity and frequency, twitching is among the most prominent of infant behaviors.

Twitching does not begin at birth. Indeed, in mammalian and avian embryos, spontaneous twitches of the distal limbs are a ubiquitous feature of behavior (Narayanan et al., 1971; Hamburger, 1973; Provine, 1973; Corner, 1977). As Corner (1977) has stated, the familiar movements that we observe in newborns during sleep are "nothing less than the continued postnatal expression of primordial nervous functional processes." In this sense, twitching is not only conspicuous, but it is developmentally and evolutionarily primitive.

\section{CHASING RABBITS}

According to the conventional or folk psychological view, described above and illustrated in the left panel of Figure 2, a dog dreaming of chasing rabbits exhibits limb movements that resemble a dog running after its prey. As the Romanes quote above attests, this view was in place long before active sleep was discovered in the middle of the last century (Aserinsky and Kleitman, 1953). Soon after that discovery, it became clear that the cortex is not the driving force behind dreaming. Rather, it is the brainstem that drives cortical activity during active sleep. It was this notion that helped inspire the activation-synthesis hypothesis of dreaming, which proposed that dreams arise from the synthesis by the cortex of internally generated signals arriving from the brainstem as well as previously stored information (Hobson and McCarley, 1977). This was an elegant theory that directly and properly challenged the hegemony of the classic Freudian interpretation of dreams.

By the time Hobson and McCarley published their activationsynthesis hypothesis, it had already been discovered that active sleep is accompanied by a profound loss of muscle tone (Pompeiano, 1967a). This loss of tone is not merely the result of muscle relaxation; rather, it results from active inhibition of the motor neurons that control the muscles. But if muscle tone is inhibited, why are not twitches inhibited as well?

The quickly accepted resolution of this apparent paradox did not stray far from the folk psychological explanation of movements during sleep. As illustrated in the middle panel of Figure 2, the dreaming 


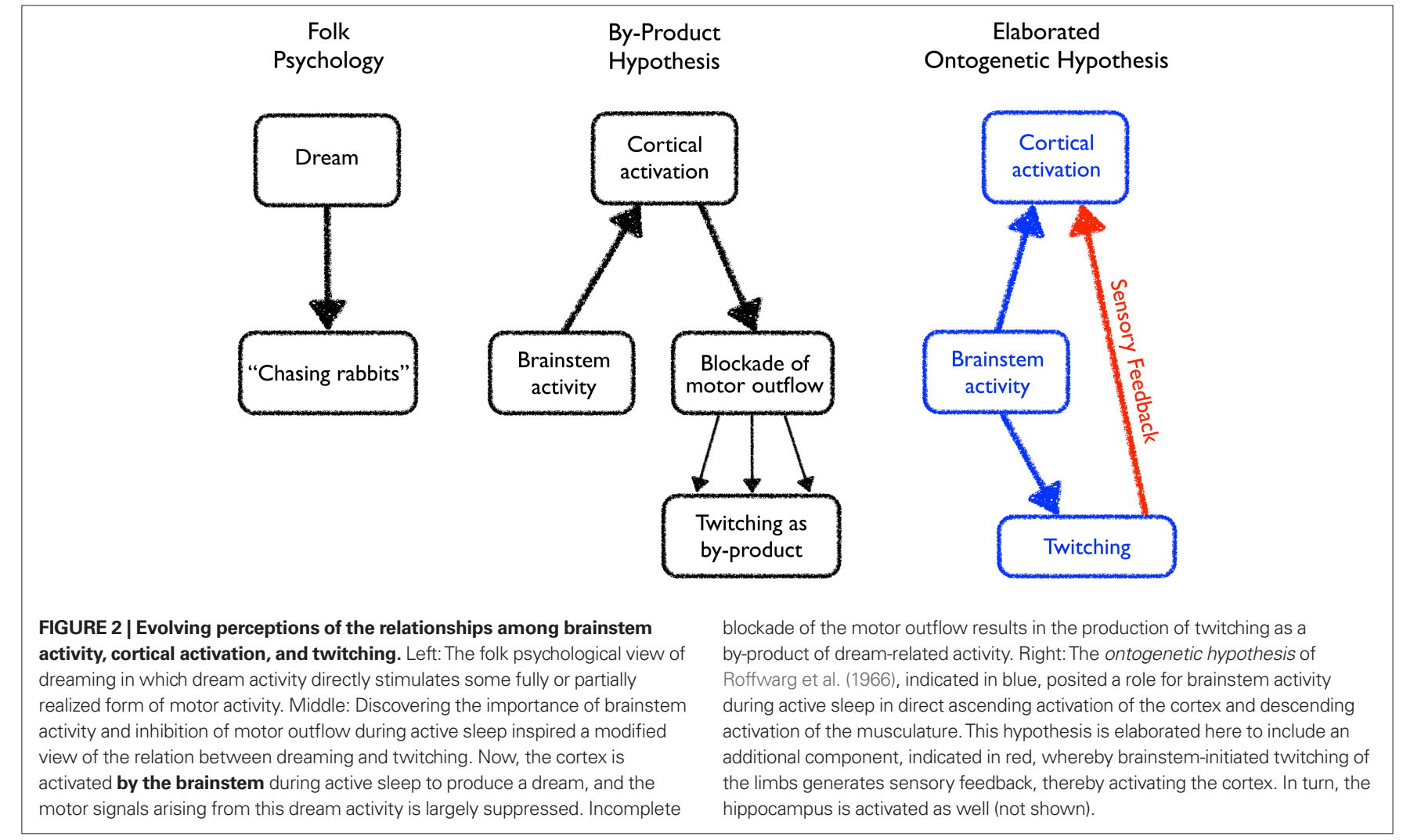

cortex was still viewed as producing movements, but now these movements were seen as mere by-products of dream activity - bits and pieces of motor commands that are able to leak through an incomplete inhibitory filter. Thus, when Hobson and McCarley presented their model relating dream activity in the cortex with movements throughout the body, they explicitly endorsed the view of twitches as imperfectly realized manifestations of movements that would be expressed as organized movements but for the inhibition of muscle tone that accompanies active sleep.

This fusing of folk psychology with sleep physiology seemed justified in light of the observation that adult cats with mesopontine lesions exhibit fully organized motor activity during active sleep - as if the cats were acting out their dreams (Jouvet and Delorme, 1965). Hobson and McCarley (1977) described this curious phenomenon like this: "The animals show all of the major manifestations of [active] sleep except the atonia; instead of the fine twitches of the digits and the limb jerks that are normally present in [active sleep], these cats display complex motor behaviors including... well-coordinated attack and defense sequences that have no apparent relationship to the environment." (p. 1337). Similarly, Chase and Morales (1990) wrote that twitches represent "brief episodes of an otherwise integrated behavior that is suppressed by the presence of motor inhibition" (p. 568, italics added). By inhibiting the integrated behavior, sleep can continue undisturbed. And as for the small twitches that break through the inhibition, they are "apparently without purpose" (p. 566).

If twitches were mere remnants of integrated behavior descending from the cortex, then we would expect that disconnecting the cortex from the brainstem would eradicate twitching. But this is not what was found. To show this, experimenters completely transected the brain into two parts. (This method has long provided valuable insights into the neural circuitry governing sleep-wake processes, beginning with Bremer's (1935) cerveau isolé preparation.) For example, when Villablanca (1966) performed transections in adult cats that prevented cortical communication with the brainstem, he reported periods of active sleep, including muscle twitches, that could not be distinguished from similar periods in non-transected cats.

Similarly, using 1-week-old rats, we performed transections that passed through or anterior to the region within the brainstem that has been implicated in the production of active sleep in adults (Kreider and Blumberg, 2000). When transections passed through this region, the expression of twitching was substantially disrupted. However, when transections were performed more rostrally such that they left the brainstem region intact but still completely disconnected the brainstem from the forebrain, the quantity and quality of twitching were completely unaffected. Subsequently, we identified neurons within the laterodorsal tegmental nucleus (LDT; a nucleus that was spared by the rostral transections discussed above) that fire in close temporal proximity to limb twitching (Karlsson et al., 2005); moreover, when electrolytic lesions were produced that included the LDT, the amount of twitching was significantly reduced despite increased amounts of sleep. All together, these findings demonstrate that twitching, in infants and adults, is not the product of dreamwork descending from the cortex.

In addition to twitching, Villablanca (1966) reported that REMs in his adult cats were unaffected by the same transections that spared limb twitching. For this and other reasons, it is worth considering the similarities between REMs and twitching. In their paper 
first reporting the discovery of REM sleep, Aserinsky and Kleitman (1953) immediately floated the possibility that REMs reflect the visual content of dreams, as if subjects watch their dreams. This scanning hypothesis is not dissimilar from the folk psychological view of limb twitching - only now the eyes are watching dreams rather than limbs acting them out. Indeed, the scanning hypothesis has itself become a part of the folk psychological interpretation of dreaming.

Although the scanning hypothesis remains popular, REMs may not provide the insight into dreaming that many have assumed. This is the view of Chase and Morales (1990), who hypothesized that "the rapid eye movements themselves can be viewed as myoclonic activity" (p. 560). According to this view, eyes are just another kind of limb and are, like the other limbs of the body, controlled by skeletal muscles that twitch during active sleep. We tested this hypothesis in infant rats by recording from the eye muscles at ages before and after the emergence of sleep-related eye movements (Seelke et al., 2005). Consistent with the Chase and Morales hypothesis, the eye muscles conformed to expectations for any skeletal muscle: the tone of the eye muscles increased during waking and decreased during sleep, and twitching of the eye muscles was detected at all ages examined - even at ages when the eyelids are sealed and the eyes do not freely move (in rats, the eyelids do not open until 15 days of age).

Thus, although the eyes have occupied a special position in the sleep literature, it may be that they are no more special than any other limb of the body. If so, then anything that we discover about the functional importance of limb twitching for the developing infant may apply with equal relevance to REMs - and vice versa.

\section{THE ONTOGENETIC HYPOTHESIS}

Howard Roffwarg was a young psychiatric resident in the early 1960s when he first began recording brain activity in young infants. Working with the pioneering sleep researcher William Dement, Roffwarg predicted that active sleep would not be detectible until 2 years of age when children are first able to talk about their dreams such was the strong link that had already been forged between active sleep and dreaming. So they were surprised to discover that not only is active sleep detectible at birth, but that the quantities of active sleep are highest at birth and even higher in preterm infants. The writing of the paper reporting these results took several years; by then they were convinced that "any hypothesis which purports to account for the regulation of REM sleep will eventually have to explain the great quantities of REM sleep during early development" (Roffwarg et al., 1966, p. 614). Their ontogenetic hypothesis was the first attempt to provide such an explanation.

In their paper, Roffwarg and colleagues turned away from dreaming to explain the great quantities of newborn active sleep. Although work over the previous decade had established a strong association between REMs and dreaming, they noted that REMs persist even in the congenitally blind as well as in humans, cats, and kittens without a functioning cortex. So they looked beyond dreaming to the brainstem activity that drives active sleep, including the forebrain activation and motor events that comprise it (see Figure 2, right panel, blue boxes and arrows). Because active sleep is so prominent in the newborn period and declines with age, and because the newborn's "waking life is limited in time and scope and offers little occasion for stimulation" (p. 614), they hypothesized that the vigorous neural stimulation arising from the brainstem during active sleep substitutes for the lack of waking stimulation. Specifically, they suggested that this sleep-related stimulation assists in a variety of developmental processes, including "neuronal differentiation, maturation, and myelinization in higher centers" (p. 616). As for the eventual emergence of dream imagery in children, they expressed a view that presaged the activation-synthesis hypothesis, which would not be formalized for another 10 years: perhaps, they wondered, "the cortex 'fits' sensory images to discharge patterns of brainstem origin"; if so, "the dream would truly appear to be born in the brainstem but clothed in the cortex" (p. 616).

The first empirical tests of the ontogenetic hypothesis, which were not performed until the 1980s, focused on the role of brainstem-generated ponto-geniculo-occipital (PGO) waves in the development of the visual system in kittens. Consistent with the ontogenetic hypothesis, PGO waves appeared to contribute to the development and neural differentiation of the lateral geniculate nucleus, a nucleus that receives visual information from the retina and transmits it to visual cortex (Davenne and Adrien, 1984, 1987; Davenne et al., 1989; Shaffery et al., 1998, 1999). Subsequent work from Roffwarg's laboratory indicates that active sleep modulates visual cortical plasticity early in development (Shaffery et al., 2002; Shaffery and Roffwarg, 2003). Significantly, these tests of the ontogenetic hypothesis have two important features in common: they focus on direct stimulation of the forebrain by the brainstem (in the form of PGO waves) and they relate exclusively to the visual system.

\section{THE ONTOGENETIC HYPOTHESIS ELABORATED}

There is no strong rationale for limiting the developmental effects of active sleep on forebrain development to either the visual system or to direct stimulation from the brainstem. Here I aim to make room within that hypothesis for twitch-related sensory feedback as a source of indirect stimulation of the forebrain. But to provide a more prominent place for twitching within the ontogenetic hypothesis and its central theme of activity-dependent brain development, we must demonstrate that sensory feedback arising from twitching limbs actually modifies brain activity. Here we immediately face an obvious problem: namely, based on decades of research, sensory inputs appear to be dampened during sleep, especially during active sleep (Pompeiano, 1967b; Taepavarapruk et al., 2002).

The phenomenon of increased sensory threshold during sleep is widespread: it has been shown using auditory (Baust et al., 1964) and olfactory (Badia et al., 1990) stimuli in adult humans, auditory (Hutt et al., 1968) and olfactory (Murray and Campbell, 1970) stimuli in human neonates, and even vibratory stimuli in flies (Shaw et al., 2000). We also reported increases in olfactory threshold during sleep in week-old rats (Seelke and Blumberg, 2004). With so much evidence consistently pointing to a diminution of sensory experience during sleep, it is perhaps unsurprising that the folk psychological interpretation of twitching as a by-product of dreaming would not have been questioned: animals may twitch all they want, but inhibition of sensory input would prevent the brain from receiving sensory information from a twitch. Until recently, there was no reason to doubt the assumption that any sensory feedback arising from twitching would be blocked or substantially diminished from entering the nervous system. 
The first strong evidence in favor of a functional role for twitches during sleep in infant rats came from Jens Schouenborg's group in Sweden (Petersson et al., 2003). Using experimental and computational approaches, they showed that tactile feedback, produced when a twitching limb touches a nearby object, guides the organization of spinal sensorimotor circuits. Thus, at least at the level of the spinal cord, sensory input provided by twitching is not blocked.

The next breakthrough came when investigators recorded from somatosensory cortex during periods of twitching in infant rats (Khazipov et al., 2004). In light of previous work that seemed to show that cortical activity was barely detectible at these early ages, the findings reported by Khazipov and colleagues were surprising. What they discovered was a new cortical event - they called it a spindle burst - comprising a brief wavelike burst of activity (a typical spindle burst might oscillate at $15 \mathrm{~Hz}$ for $1 \mathrm{~s}$ ). Even more surprising was that these spindle bursts were triggered by twitches during sleep in a topographic manner: forelimb twitches triggered spindle bursts in the forelimb region of somatosensory cortex; hindlimb twitches triggered spindle bursts in the hindlimb region, etc. That the causal arrow flowed from limb twitch to cortex was established by transecting the spinal cord, thereby blocking the flow of sensory information from the hindlimbs, and effectively decreasing the number of spindle bursts produced. Subsequent work from this group demonstrated that spindle bursts also occur in visual cortex in response to retinal waves (Hanganu et al., 2006) and in barrel cortex in response to whisker movements (Minlebaev et al., 2007). Thus, this newly discovered cortical event - perhaps the most prominent cortical event in the developing cortex - may play a critical role in organizing cortical circuits involved in the processing of sensory information.

We further explored the relationship between spindle bursts and sensory processing using an experimental paradigm that allowed us to observe twitching and also deliver discrete sensory stimulation to the forepaw (Marcano-Reik and Blumberg, 2008). In these experiments, we simultaneously recorded from the region of somatosensory cortex that processes information from the forepaw. First, we found that spindle bursts could be reliably evoked by forepaw stimulation during sleep or during wakefulness. Second, we found that stimulating the forepaw in such a way as to produce tactile stimulation of the plantar surface and dorsiflexion of the wrist - that is, touching and flexing - produced a spindle burst that was superimposed within a second cortical event that we called a slow potential. We were able to dissociate the two events by stroking the plantar surface alone - thereby producing only the slow potential - or by flexing the wrist alone - thereby producing only the spindle burst. Accordingly, we suggested that limb movements during twitching produce proprioceptive feedback that triggers spindle bursts; in contrast, the slow potential would only be produced when a twitching limb makes contact with another object (e.g., a littermate or mother).

Although we now know that twitching in early development drives cortical activity, we do not yet have a sense of the magnitude of this activity. In another developmental domain - the emergence of locomotion in human infants - a compelling argument has been made about the formative role of experience by simply documenting the sheer quantity of locomotor activity that infant crawlers and walkers exhibit (Adolph, 2005). For example, each day an infant crawler covers, in short bursts, the lengths of two football fields. In light of such evidence, the formative role of locomotor experience is difficult to ignore. We can take a similar approach with twitching.

We routinely record activity in the nuchal muscle - the muscle located at the back of the neck that is responsible for holding our head upright. From these recordings we can count the number of twitches that occur. When we did this, we estimated that this one muscle twitches over 38,000 times each day (Marcano-Reik et al., 2010). Because we were recording cortical activity in these same animals, we were able to similarly quantify the number of spindle bursts: we estimated that within one discrete region of somatosensory cortex, 4,000 spindle bursts are generated each day. This extraordinary level of activity is hard to dismiss.

As shown in the right panel of Figure 2 and elaborated in Figure 3, a fresh perspective has emerged that departs substantially from the dominant view that has guided our thinking about twitching until now. With the addition of sensory feedback, twitching is potentially transformed from a functionless by-product of dreaming to an active contributor to sensorimotor integration within the spinal cord and brain, including the development, refinement, and maintenance of somatotopic maps. Indeed, it may be that twitching contributes to the functional linking of muscle and somatosensory cortex in a way that is similar to how retinal waves contribute to the linking of retina and visual cortex (Katz and Shatz, 1996; Wong, 1999). This notion of activity-dependent development was at the heart of Roffwarg et al.'s ontogenetic hypothesis, and so the view of twitching promoted here should be considered an elaboration of that hypothesis.

In support of a functional role for twitching, we were recently able to document a relationship among twitching, spindle burst production, and the plasticity of somatosensory cortex (MarcanoReik et al., in press). To demonstrate a change in plasticity, we relied on a previous finding that transecting the corpus callosum - the large bundle of fibers that connects the two cerebral hemispheres - immediately decreases the reliability with which spindle bursts occur in response to forepaw stimulation, from nearly $100 \%$ to $70 \%$ (Marcano-Reik and Blumberg, 2008). In our subsequent study, we again transected the corpus callosum, but now we assessed recovery of function over the ensuing week. We found that recovery of function occurred only if the corpus callosum was transected at or before 6 days of age. After that age, recovery of function did not occur (in fact, the reliability of responding worsened), thus indicating a reduction in cortical plasticity. Also around 6 days of age, we noted several other dramatic developmental changes, including an abrupt transition in how the corpus callosum modulates spindle bursts, a rapid increase in intrinsic cortical synaptic connectivity, and a transformation in GABA's functional properties. That these changes occur contemporaneously with an abrupt decrease in the rates of twitching provides further support for the hypothesis that this sleep-related behavior participates in the activity-dependent development and refinement of cortical networks.

The functional consequences of twitching for the developing brain may not end with the cortex. In fact, the hippocampus also exhibits activity patterns that are strongly linked with twitching (Mohns and Blumberg, 2008). Recording from the hippocampus of 1- to 


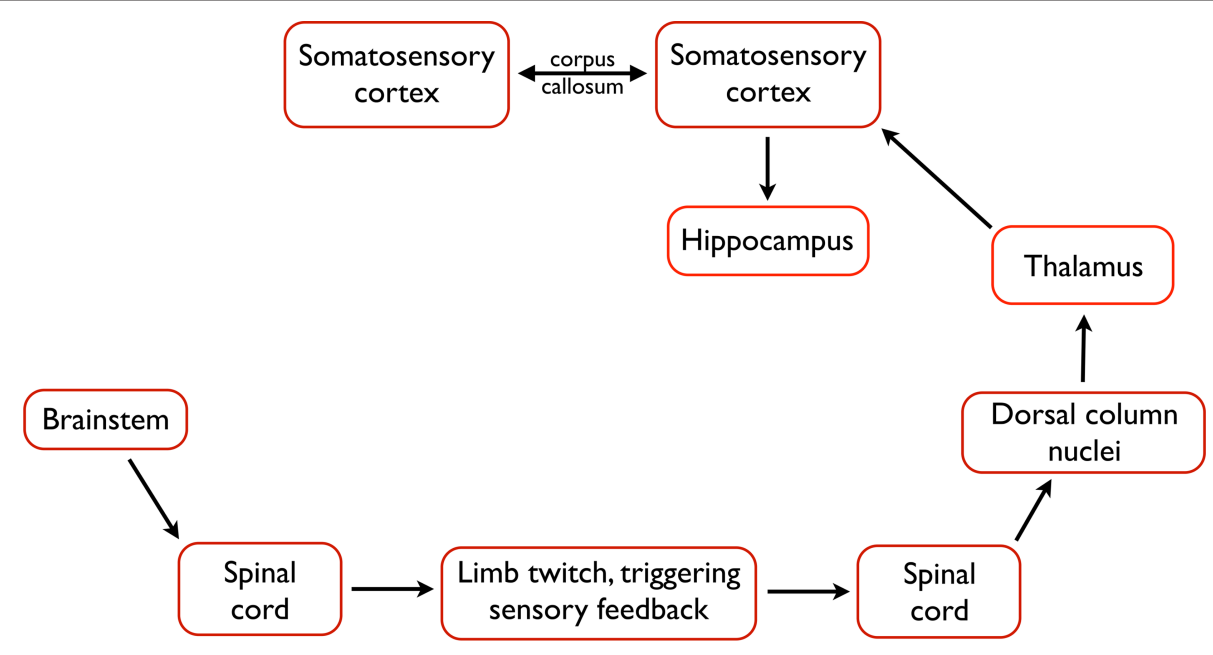

FIGURE 3 |The likely pathway from the triggering of a twitch to the processing of twitch-related sensory feedback in the forebrain. Neurons within the brainstem trigger a limb twitch, whereupon sensory feedback, including proprioceptive feedback, is generated. Proprioceptive feedback is communicated through the spinal cord, dorsal column nuclei, and thalamus before generating a spindle burst in primary somatosensory cortex. From the cortex, activation is communicated to the hippocampus and, perhaps, to other structures. In addition, interactions between homotopic regions of left and right somatosensory cortex, via the corpus callosum, modulate spindle burst activity. 12-day-old rats, we found that the majority of hippocampal neurons increase their firing rates during periods of myoclonic twitching of the limbs, and a subset of these neurons exhibit a burst of activity immediately after a limb twitch, again suggesting that the twitches themselves provide sensory feedback to the infant hippocampus. We next showed that disconnecting the cortex and hippocampus had no effect on the production of spindle bursts but eliminated twitch-related activity in the hippocampus, thus revealing the path of activation from cortex to hippocampus (Mohns and Blumberg, 2010). We do not yet know whether twitch-related activation of the brain continues beyond the hippocampus. But for now, we propose that twitching not only contributes to the development and refinement of neural circuits within cortex and hippocampus, but also helps coordinate interactions between those two structures, whose integrated relations are critical for learning and memory.

\section{IS TWITCHING PERMISSIVE OR INSTRUCTIVE?}

How can we be sure that twitching per se is important for central nervous system development? After all, cortical spindle bursts occur during waking movements such as kicking and stretching. At this time, we can only outline several possibilities.

It is a fact of mammalian development that twitching is associated with active sleep and active sleep occupies the plurality of time of the young infant. By contrast, early in development, wakefulness and its associated behaviors are relatively rare. For this reason, Roffwarg et al. (1966) hypothesized that active sleep can be interpreted as a means of stimulating the brain in the absence of waking stimulation. According to this view, active sleep provides a substitute for the stimulation that wakefulness will provide later in life.

The notion that stimulation of any kind - during sleep or waking - may be sufficient to drive activity-dependent processes raises the key distinction between permissive and instructive roles of activity in brain development (Crair, 1999). When neural activity has permissive effects, it only matters that some form of activity occurs. Accordingly, if twitching played a permissive role in brain development, the temporal and spatial qualities of a twitch or the fact that it happens during active sleep would not matter. In this broad sense of permissive, all that would be needed for the development of sensorimotor integration is some form of activation, regardless of its source or patterning. If so, then perhaps twitching is favored over waking movements only because twitching stimulates the brain without arousing the infant.

In the visual system, long before animals experience patterned visual input, rhythmic bursting activity within each retina contributes to the development and refinement of connections among retina, thalamus, and visual cortex (Katz and Shatz, 1996; Wong, 1999). Because the source and patterning of these retinal waves influence the kinds of downstream effects observed, they are viewed as playing an instructive role in visual system development (Crair, 1999). The question we face is whether twitching plays such an instructive role in the development of the sensorimotor system.

There are several features of twitching that may contribute to its playing an instructive role. First, if twitching were to help establish precise somatotopic relations between muscle and brain, then it would be important that each twitch movement be discrete. We have shown that twitch movements in rat fetuses and newborns are discrete, rarely if ever occurring simultaneously with twitches in other limbs (Robinson et al., 2000). In contrast, waking movements - including stretching and kicking - are not only larger movements involving many muscle groups within a limb, but they also often involve simultaneous movements in more than one limb. Thus, just as retinal waves contribute to the segregation of inputs from the two eyes, twitches may help to segregate inputs from separate muscle groups within a limb and between different limbs.

Second, twitches occur during active sleep when muscle tone is low. As a consequence, sensory feedback from small movements will be easier to detect during active sleep (i.e., the signal-to-noise ratio will be higher). When muscle tone increases during wakefulness, 
background noise will also increase, thereby obscuring sensory feedback arising from relatively small movements. Thus, the temporal patterning of twitching and its occurrence during periods of low muscle tone may work together to ensure that the nervous system establishes clearer associations between motor outputs and sensory inputs.

Finally, active sleep may be a behavioral state that is conducive to neuroplasticity. If so, then twitching may promote neural development in part because of its association with active sleep. For example, acetylcholine (ACh) release from the basal forebrain of adults is highest during active sleep (Vazquez and Baghdoyan, 2001) and ACh is an established modulator of cortical plasticity (Bear and Singer, 1986; Rasmusson and Dykes, 1988; Tremblay et al., 1990; Juliano et al., 1991; Kilgard and Merzenich, 1998; Rasmusson, 2000). Thus, it is possible that active sleep, through release of cortical ACh, sets the stage for twitching to modify somatosensory circuits.

Efference copy is a well-established mechanism by which animals distinguish self-produced movements from sensations from movements evoked by exogenous stimuli (Blakemore et al., 2000; Cullen, 2004). Accordingly, in addition to the caudally projecting signal that results in a twitch, there may be a rostrally projecting signal that "prepares" the nervous system for the ensuing arrival of twitch-related sensory information, thereby contributing to sensorimotor development and somatotopic organization. This efference copy could be transmitted to the cholinergic basal forebrain, which could then modulate somatosensory cortex in anticipation of the ensuing arrival of sensory information. Indeed, in infant rats, ACh released from the basal forebrain contributes to spindle burst production in visual cortex (Hanganu et al., 2007).

\section{REM BEHAVIOR DISORDER (RBD) AND THE ACTIVATION-SYNTHESIS HYPOTHESIS REVISITED}

The historical progression of ideas illustrated in Figure 2 may also help us make sense of other sleep-related phenomena. For example, REM behavior disorder (RBD) is a degenerative disease that affects mostly older men and has a prevalence of approximately $0.5 \%$ (Fantini et al., 2005). As with the cats with mesopontine lesions described earlier, individuals with RBD enter active sleep but, instead of exhibiting the normal features of muscle atonia and twitching, they behave in ways that suggest they are "acting out their dreams" (Mahowald and Schenck, 2005). These behaviors are many and varied: they include yelling, grabbing, punching, and kicking. The dreams of RBD patients are reported to be as aggressive as these behaviors.

Subsequent analyses of cats with mesopontine lesions called into question the enticing idea that they were acting out their dreams (Morrison, 1983). Instead, it became clear that these lesions were not only preventing muscle atonia, they were also disinhibiting locomotor drive. But if these cats were not acting out their dreams, how might we account for the relation between the reported dreams of RBD patients and their "dream-enactment" behaviors?

The conventional interpretation of RBD seems to fit well with the scheme presented in the middle panel of Figure 2: brainstem activity drives cortical activity, dreams are produced, and the absence of motor inhibition in RBD allows the motor features of dreams to be implemented without interference. But what if this view gets it backward? What if the right panel of Figure 2 comes closer to explaining the dream-behavior relationship in RBD? That is, given that brainstem activity during active sleep drives motor activity, the damaged brainstems of RBD patients may result in the production of more robust sleep-related behaviors. Then, the sensory feedback from these vigorous movements could trigger the violent dreams of RBD. Consistent with this view, Fantini et al. (2005) suggest that the increase in sleep-related motor activity in RBD "may be responsible for both motor behaviors and actionfilled dreams" (p. 1014).

Fantini and colleagues view their inversion of the dreambehavior causal sequence in RBD as perfectly consonant with the activation-synthesis hypothesis of dreaming. I agree, although it should be noted that Hobson and McCarley (1977) do not appear to have envisioned a role for sensory feedback in the modulation of dream content. Still, just as the ontogenetic hypothesis can incorporate sensory feedback from twitching while still retaining its central theme of activity-dependent development during sleep, the activation-synthesis model can incorporate an unanticipated form of cortical activation. Indeed, given that sleepers often incorporate sensory information into dreams (Dement and Wolpert, 1958; Berger, 1963), can we imagine that patients with RBD would fail to incorporate their own yelling, grabbing, punching, and kicking into their dreams?

\section{CONCLUSIONS AND FUTURE DIRECTIONS}

Each infant faces the challenge of adapting and functioning in the moment even as its body grows, limbs elongate, muscles strengthen, brain differentiates and forms new connections, and ecological niches and social interactions change (Alberts and Cramer, 1988). Such dramatic shifts and upheavals necessitate flexible developmental systems. Preprogrammed and prescribed solutions will not do because the problems to be solved around the next bend cannot be foreseen. This principle applies to all of us, whether typically or atypically formed (Blumberg, 2009). It is in this context that we may be able to make sense of the great quantities of sleep in newborns. And so it is in this context that the ontogenetic hypothesis retains its force.

Although twitching has long been viewed as by-products of a dreaming brain, we now have ample reason to move on to new ideas. Of course, we might have questioned the by-product hypothesis on the simple grounds that so much twitching in a developing infant wastes energy that would be more wisely devoted to growth. But that argument alone could never have displaced the supremacy of the by-product hypothesis.

That spontaneous activity plays a critical role in the development of the nervous system is now a foundational concept in the field (Shatz, 1990; Purves, 1994; O’Donovan, 1999). And can there be any more conspicuous and dramatic example of spontaneous activity than twitching in newborns? Of course, it is possible that all this twitching might have had no effect on neural activity. Indeed, this possibility was assumed to be true because of the well-known increase in sensory thresholds that accompanies sleep. However, it is now clear that sleep-related increases in sensory threshold do not prevent the feedback from these self-generated movements from entering the central nervous system. On the contrary, sensory 
feedback from twitching substantially modifies neural activity from the spinal cord to the cortex to the hippocampus. Thus, we are now able to glimpse like never before how infant sleep may contribute to developmental flexibility and evolutionary change (Blumberg and Seelke, 2010).

It is important to determine whether twitching plays a permissive or instructive role in development. But beyond that issue we will still have much to learn. In addition to its effects on neural development, twitch movements may influence the development of bone, cartilage, and muscle (Blumberg and Lucas, 1996; Müller, 2003). Also, although twitching does not end in infancy, we do not yet have a clear picture of the quality and quantity of twitching across the lifespan or in different species, including humans. We also do not know if the adolescent or adult brain experiences the sensory feedback from twitching as infants do.

This new view of twitching should open new and potentially fruitful avenues for basic research and clinical application. For example, twitches may prove to be sensitive and powerful tools for plotting the trajectory of normal and abnormal somatosensory development across the lifespan; for diagnosing sensorimotor dysfunction and recovery of function after peripheral injury and brain damage; and for assessing the effectiveness of therapies designed to improve sensorimotor function after injury. Perhaps most intriguing is the possibility that the contributions of twitching to somatosensory plasticity in normal development are recapitulated during recovery from stroke or brain injury, or are recruited as amputees learn to rely more heavily on their remaining limbs.

Important clues to the relations between sleep-related motor activity and neuropathology are already emerging. Consider once again $\mathrm{RBD}$, a neurodegenerative disorder characterized by disruption of the brainstem mechanisms that normally inhibit motor activity during active sleep. Interestingly, clinical investigators have been developing increasingly precise and quantitative measures of twitching with the hope that such measures will facilitate improved understanding of the neurological deficits in RBD (Lapierre and Montplaisir, 1992; Bliwise et al., 2006; Frauscher

\section{REFERENCES}

Adolph, K. E. (2005). "Learning to learn in the development of action," in Action as an Organizer of Perception and Cognition During Learning and Development: Minnesota Symposium on Child Development, Vol. 33, eds, J. Lockman, J. Reiser and C. A. Nelson (Hillsdale, NJ: Erlbaum), 91-122.

Alberts, J. R., and Cramer, C. P. (1988). "Ecology and experience: sources of means and meaning of developmental change," in Handbook of Behavioral Neurobiology, Vol. 8, ed M. Blass (New York: Plenum Press), 1-39.

Aserinsky, E., and Kleitman, N. (1953). Regularly occurring periods of eye motility, and concomitant phenomena, during sleep. Science 118 , 273-274.

Aton, S. J., Seibt, J., Dumoulin, M., Jha, S. K., Steinmetz, N., Coleman, T.,

et al., 2008; Iranzo et al., 2009). For example, although investigators typically emphasize the "dream-enactment" aspects of RBD, twitching in these patients increases during active sleep - even on nights when overt behavioral abnormalities are not expressed (Bliwise and Rye, 2008).

As surprising as RBD is, even more surprising is that RBD patients are at much greater risk of developing Parkinson's Disease and other related disorders (Schenck et al., 1996). In fact, RBD can precede the onset of these other disorders by many decades (Claassen et al., 2010). In other words, a dysfunction of motor activity and muscle control during active sleep has now been associated with a devastating degenerative motor disease of old age. Moreover, this association is made even stronger by observations, in Parkinson's patients, of increased twitching during sleep (Bliwise et al., 2010) and even evidence of "restored" motor control during sleep (De Cock et al., 2007). Such observations suggest that systematic and precise measures of twitching in humans could prove useful for the diagnosis, treatment, and, perhaps someday, prevention of these and other neuropathological conditions.

It is difficult to foresee all possible directions for this research because so little is known about twitching across the lifespan in health and disease. Normative data are needed to provide a basis for comparison with pathological and injured populations. Then we will be in a better position to generate hypotheses and test them in humans and other animals. Ultimately, by seriously considering twitching as a complex behavior in its own right, we may someday come to appreciate that these seemingly inconsequential movements are more meaningful than anyone ever dreamed.

\section{ACKNOWLEDGMENTS}

Preparation of this article was made possible by a research grant (MH50701) and an Independent Scientist Award (MH66424) from the National Institute of Mental Health. I thank Howard Roffwarg, James Shaffery, William Todd, Amy Jo Marcano-Reik, and Andy Gall for helpful comments on an earlier draft of the manuscript. I also thank Howard Roffwarg for sharing his recollections with me about the origins and early days of the ontogenetic hypothesis.

Blakemore, S. J., Wolpert, D., and Frith, C. (2000). Why can't you tickle yourself? Neuroreport 11, R11-R16.

Bliwise, D. L., He, L., Ansari, F. P., and Rye, D. B. (2006). Quantification of electromyographic activity during sleep: a phasic electromyographic metric. J. Clin. Neurophysiol. 23, 59-67.

Bliwise, D. L., and Rye, D. B. (2008) Elevated PEM (phasic electromyographic metric) rates identify rapid eye movement behavior disorder patients on nights without behavioral abnormalities. Sleep 31 , 853-857.

Bliwise, D. L., Trotti, L. M., Greer, S. A., Juncos, J. J., and Rye, D. B. (2010). Phasic muscle activity in sleep and clinical features of Parkinson disease. Ann. Neurol. 68, 353-359.

Blumberg, M. S. (2009). Freaks of Nature: What Anomalies Tell Us about
Development and Evolution. New York: Oxford University Press.

Blumberg, M.S., and Lucas, D. E. (1996). A developmental and component analysis of active sleep. Dev. Psychobiol. 29, $1-22$.

Blumberg, M. S., and Seelke, A. M. (2010). "The form and function of infant sleep: from muscle to neocortex," in The Oxford Handbook of Developmental Behavioral Neuroscience, eds M. S. Blumberg, J. H. Freeman, and S.R. Robinson (New York: Oxford University Press), 391-423.

Bremer, F. (1935). Cerveau "isole" et physiologie du sommeil. Comp. Rendus Soc. Biol. 118, 1235-1241.

Chase, M. H., and Morales, F. R. (1990). The atonia and myoclonia of active (REM) sleep. Annu. Rev. Psychol. 41, 557-584.

Claassen, D. O., Josephs, K. A., Ahiskog, J. E., Silver, M. H., Tippmann-Peikert, 
M., and Boeve, F. F. (2010). REM sleep behavior disorder preceding other aspects of synucleinopathies by up to half a century. Neurology 75, 494-499.

Corner, M.A. (1977). Sleep and the beginnings of behavior in the animal kingdom - studies of ultradian motility cycles in early life. Prog. Neurobiol. 8, 279-295.

Crair, M. C. (1999). Neuronal activity during development: permissive or instructive? Curr. Opin. Neurobiol. 9, 88-93.

Cullen, K. E. (2004). Sensory signals during active versus passive movement. Curr. Opin. Neurobiol. 14, 698-706.

Davenne, D., and Adrien, J. (1984). Suppression of PGO waves in the kitten: anatomical effects on the lateral geniculate nucleus. Neurosci. Lett. 45, 33-38.

Davenne, D., and Adrien, J. (1987). Lesion of the ponto-geniculo-occipital pathways in kittens. I. Effects on sleep and on unitary discharge of the lateral geniculate nucleus. Brain Res. 409, $1-9$.

Davenne, D., Fregnac, Y., Imbert, M., and Adrien, J. (1989). Lesion of the PGO pathways in the kitten. II. Impairment of physiological and morphological maturation of the lateral geniculate nucleus. Brain Res. 485, 267-277.

De Cock, V. C., Vidailhet, M., Leu, S., Texeira, A., Apartis, E., Elbaz, A., Roze, E., Willer, J. C., Derenne, J. P., Agid, Y., and Arnulf, I. (2007). Restoration of normal motor control in Parkinson's disease during REM sleep. Brain 130, 450-456.

Dement, W. C., and Wolpert, E. (1958). The relation of eye movements, body motility, and external stimuli to dream content. J. Exp. Psychol. 55, 543-553.

Fantini, M. L., Corona, A., Clerici, S., and Ferini-Strambi, L. (2005). Aggressive dream content without daytime aggressiveness in REM sleep behavior disorder. Neurology 65, 1010-1015.

Fantini, M. L., Ferini-Strambi, L., and Montplaisir, J. (2005). Idiopathic REM sleep behavior disorder: toward a better nosologic definition. Neurology 64 , 780-786.

Fifer, W. P., Byrd, D. L., Kaku, M., Eigsti, I. M., Isler, J. R., Grose-Fifer, J., Tarullo, A. R., and Balsam P. D. (2010). Newborn infants learn during sleep. Proc. Natl. Acad. Sci. U.S.A. 107, 10320-10323.

Frank, M. G., Issa, N. P., and Stryker, M. P. (2001). Sleep enhances plasticity in the developing visual cortex. Neuron 30, 275-287.

Frauscher, B., Iranzo, A., Hogl, B., Casanova-Molla, J., Salamero, M., Gschliesser, V., Tolosa, E., Poewe, W., Santamaria, J., and SINBAR. (2008). Quantification of electromyographic activity during REM sleep in multiple muscles in REM sleep behavior disorder. Sleep 31, 724-731.

Gramsbergen, A., Schwartze, P., and Prechtl, H. F. R. (1970). The postnatal development of behavioral states in the rat. Dev. Psychobiol. 3, 267-280.

Hamburger, V. (1973). "Anatomical and physiological bases of embryonic motility in birds and mammals," in Studies on the Development of Behavior and the Nervous System, Behavioral Embryology, Vol. 1, ed G. Gottlieb (New York: Academic Press), 51-76.

Hanganu, I. L., Ben-Ari, Y., and Khazipov, R. (2006). Retinal waves trigger spindle bursts in the neonatal rat visual cortex. J. Neurosci. 26, 6728-6736.

Hanganu, I. L., Staiger, J. F., Ben-Ari, Y., and Khazipov, R. (2007). Cholinergic modulation of spindle bursts in the neonatal rat visual cortex in vivo. $J$. Neurosci. 27, 5694-5705.

Hobson, J.A., and McCarley, R. W. (1977). The brain as a dream state generator: an activation-synthesis hypothesis of the dream process. Am. J. Psychiatry 134, 1335-1348.

Holtzman, D., Obana, K., and Olson, J. (1981). Hyperthermia-induced seizures in the rat pup: a model for febrile convulsions in children. Science 213, 1034-1036.

Hupbach, A., Gomez, R. L., Bootzin, R. R., and Nadel, L. (2009). Nap-dependent learning in infants. Dev. Sci. 12, 1007-1012.

Hutt, S. J., Hutt, C., Lenard, H. G., von Bernuth, H., and Muntjewerff, W. J. (1968). Auditory sensitivity in the human neonate. Nature 218, 888-890.

Iranzo, A., Luca Ratti, P., CasanovaMolla, J., Serradell, M., Vilaseca, I., and Santamaria, J. (2009). Excessive muscle activity increases over time in idiopathic REM sleep behavior disorder. Sleep 32, 1149-1153.

Jouvet, M., and Delorme, F. (1965). Locus coeruleus et sommeil paradoxal. Comp Rendus Soci. Biol. 159, 895-899.

Jouvet-Mounier, D., Astic, L., and Lacote, D. (1970). Ontogenesis of the states of sleep in rat, cat, and guinea pig during the first postnatal month. Dev. Psychobiol. 2, 216-239.

Juliano, S. L., Ma, W., and Eslin, D. (1991). Cholinergic depletion prevents expansion of topographic maps in somatosensory cortex. Proc. Natl. Acad. Sci.U.S.A. 88, 780-784

Karlsson, K. Æ., Gall, A. J., Mohns, E. J., Seelke, A. M. H., and Blumberg, M. S (2005). The neural substrates of infant sleep in rats. PLoS Biol. 3, 891-901. doi: 10.137/joumal.pbio.0030143.

Katz, L. C., and Shatz, C. J. (1996). Synaptic activity and the construction of cortical circuits. Science 274, 1133-1138.
Khazipov, R., Sirota, A., Leinekugel, X., Holmes, G. L., Ben-Ari, Y., and Buzsaki, G. (2004). Early motor activity drives spindle bursts in the developing somatosensory cortex. Nature 432, 758-761.

Kilgard, M. P., and Merzenich, M. M. (1998). Cortical map reorganization enabled by nucleus basalis activity. Science 279, 1714-1718.

Korman, M., Doyon, J., Doljansky, J., Carrier, J., Dagan, Y., and Karni, A (2007). Daytime sleep condenses the time course of motor memory consolidation. Nature Neurosci. 10 1206-1213.

Kreider,J.C., and Blumberg, M. S. (2000). Mesopontine contribution to the expression of active 'twitch' sleep in decerebrate week-old rats. Brain Res. 872, 149-159.

Lapierre, O., and Montplaisir, J. (1992). Polysomnograhic features of REM sleep behavior disorder: development of a scoring method. Neurology 42, 1371-1374.

Mahowald, M. W., and Schenck, C. H. (2005). Insights from studying human sleep disorders. Nature 437 1279-1285.

Marcano-Reik, A. J., and Blumberg, M. S. (2008). The corpus callosum modulates spindle-burst activity within homotopic regions of somatosensory cortex in newborn rats. Eur. J. Neurosci. 28, 1457-1466.

Marcano-Reik, A. J., Prasad, T., Weiner,J.A. and Blumberg, M. S. (2010). An abrupt development shift in callosal modulation of sleep-related spindle bursts coincides with the emergence of excitatory-inhibitory balance and a reduction of somatosensory cortical plasticity. Behav. Neurosci.124, 600-611.

Margoliash, D. (2005). Song learning and sleep. Nat. Neurosci. 8, 546-548.

Millan, C. (2009). Canine sleep. from http://www.cesarsway.com/askcesar/ aboutcesar-canine-sleep.

Minlebaev, M., Ben-Ari, Y., and Khazipov, R. (2007). Network mechanisms of spindle-burst oscillations in the neonatal rat barrel cortex in vivo. $J$. Neurophysiol. 97, 692-700.

Mohns, E. J., and Blumberg, M. S. (2008). Synchronous bursts of neuronal activity in the developing hippocampus: modulation by active sleep and association with emerging gamma and theta rhythms. J. Neurosci. 28 10134-10144.

Mohns, E. J., and Blumberg, M. S. (2010) Neocortical activation of the hippocampus during sleep in newborn rats. J. Neurosci. 30, 3438-3449.

Morrison, A. R. (1983). A window on the sleeping brain. Sci. Am. 248, 94-102.

Müller, G. B. (2003). Embryonic motility: environmental influences and evolutionary innovation. Evol. Dev. 5, 56-60.

Murray, B., and Campbell, D. (1970). Differences between olfactory thresholds in two sleep states in the newborn infants. Psychon. Sci. 18, 313-314.

Narayanan, C. H., Fox, M. W., and Hamburger, V. (1971). Prenatal development of spontaneous and evoked activity in the rat (Rattus norvegicus). Behaviour 40, 100-134.

O'Donovan, M. J. (1999). The origin of spontaneous activity in developing networks of the vertebrate nervous system. Curr. Opin. Neurobiol. 9, 94-104.

Oksenberg, A., Shaffery, J. P., Marks, G. A., Speciale, S. G., Mihailoff, G., and Roffwarg, H. P. (1996). Rapid eye movement sleep deprivation in kittens amplifies LGN cell-size disparity induced by monocular deprivation. Dev. Brain Res. 97, 51-61.

Petersson, P., Waldenström, A., Fåhraeus, C., and Schouenborg, J. (2003). Spontaneous muscle twitches during sleep guide spinal self-organization. Nature 424, 72-75.

Pompeiano, O. (1967a). The neurophysiological mechanisms of the postura and motor events during desynchronized sleep. Res. Publ. Ass. Res. Nerv. Men. Dis. 45, 351-423.

Pompeiano, O. (1967b). "Sensory inhibition during motor activity in sleep," in Neurophysiological Basis of Normal and Abnormal Motor Activities, eds M. D. Yah and D. P. Purpura (Hewlett, NY: Raven Press), 323-375.

Provine, R.R.(1973). “Neurophysiological aspects of behavior development in the chick embryo" in Behavioral Embryology, Vol. 1 (New York: Academic Press), pp. 77-102.

Purves, D. (1994). Neural Activity and the Growth of the Brain. Cambridge: Cambridge University Press.

Rasmusson, D. D. (2000). The role of acetylcholine in cortical synaptic plasticity. Behav. Brain Res. 115 , 205-218.

Rasmusson,D.D., and Dykes, R.W. (1988) Long-term enhancement of evoked potentials in cat somatosensory cortex produced by co-activation of the basal forebrain and cutaneous receptors. Exp. Brain Res. 70, 276-286.

Robinson, S. R., Blumberg, M. S., Lane, M. S., and Kreber, L. A. (2000). Spontaneous motor activity in fetal and infant rats is organized into discrete multilimb bouts. Behav. Neurosci. 14, 328-336.

Roffwarg, H. P., Muzio, J. N., and Dement, W. C. (1966). Ontogenetic development of the human sleep-dream cycle. Science 152, 604-619.

Romanes, G. J. (1883/1977). Animal Intelligence. Washington, DC: 
University Publications of American, Inc.

Schenck, C. H., Bundlie, S. R., and Mahowald, M. W. (1996). Delayed emergence of a parkinsonian disorder in $38 \%$ of 29 older men initially diagnosed with idiopathic rapid eye movement sleep behaviour disorder. Neurology 46, 388-393.

Seelke, A. M. H., and Blumberg, M. S. (2004). Sniffing in infant rats during sleep and wakefulness. Behav. Neurosci. 118, 267-273.

Seelke, A. M. H., and Blumberg, M. S. (2005). Thermal and nutritional modulation of sleep in infant rats. Behav. Neurosci. 19, 603-611.

Seelke, A. M. H., and Blumberg, M. S. (2008). The microstructure of active and quiet sleep as cortical delta activity emerges in infant rats. Sleep 31, 691-699.

Seelke, A. M. H., Karlsson, K. Æ., Gall, A. J., and Blumberg, M. S. (2005). Extraocular muscle activity, rapid eye movements, and the development of active and quiet sleep. Eur. J.Neurosci. 22, 911-920.

Shaffery, J. P., Oksenberg, A., Marks, G. A., Speciale, S. G., Mihailoff, G., and
Roffwarg, H. P. (1998). REM sleep deprivation in monocularly occluded kittens reduces the size of cells in LGN monocular segment. Sleep 21, 837-845.

Shaffery, J. P., and Roffwarg, H. P. (2003). Rapid eye-movement sleep deprivation does not 'rescue' developmentally regulated long-term potentiation in visual cortex of mature rats. Neurosci. Lett. 342, 196-200.

Shaffery, J. P., Roffwarg, H. P., Speciale, S. G., and Marks, G. A. (1999). Pontogeniculo-occipital-wave suppression amplifies lateral geniculate nucleus cell-size changes in monocularly deprived kittens. Dev. Brain Res. 114, 109-119.

Shaffery, J. P., Sinton, C. M., Bissette, G., Roffwarg, H.P., and Marks, G.A. (2002). Rapid eye movement sleep deprivation modifies expression of long-term potentiation in visual cortex of immature rats. Neuroscience 110, 431-443.

Shatz, C. J. (1990). Impulse activity and the patterning of connections during CNS development. Neuron 5, 745-756.

Shaw, P. J., Cirelli, C., Greenspan, R. J., and Tononi, G. (2000). Correlates of sleep and waking in Drosophila melanogaster. Science 287, 1834-1837.

Siegel, J. M. (2001). The REM sleepmemory consolidation hypothesis. Science 294, 1058-1063.

Smith, C. (1995). Sleep states and memory processes. Behav. Brain Res. 69 , 137-145.

Stickgold, R. (2005). Sleep-dependent memory consolidation. Nature 437, 1272-1278.

Taepavarapruk, N., McErlane, S. A., and Soja, P. J. (2002). State-related inhibition by GABA and glycine of transmission in Clarke's column. J. Neurosci. 22, 5777-5788.

Tremblay, N., Warren, R. A., and Dykes, R. W. (1990). Electrophysiological studies of acetylcholine and the role of the basal forebrain in the somatosensory cortex of the cat. II. Cortical neurons excited by somatic stimuli. J. Neurophysiol. 64, 1212-1222.

Vazquez, J., and Baghdoyan, H.A. (2001). Basal forebrain acetylcholine release during REM sleep is significantly greater than during waking. Am. J. Physiol. 280, R598-R601.

Villablanca, J. (1966). Behavioral and polygraphic study of "sleep" and "wakefulness" in chronic decerebrate cats. Electroencephalogr. Clin. Neurophysiol. 21, 562-577.

Wong, R. O. (1999). Retinal waves and visual system development. Annu. Rev. Neurosci. 22, 29-47.

Conflict of Interest Statement: The author declares that the research was conducted in the absence of any commercial or financial relationships that could be construed as a potential conflict of interest.

Received: 21 August 2010; accepted: 08 October 2010; published online: 01 November 2010.

Citation: Blumberg MS (2010) Beyond dreams: do sleep-related movements contribute to brain development? Front. Neur. 1:140. doi: 10.3389/fneur.2010.00140

This article was submitted to Frontiers in Sleep and Chronobiology, a specialty of Frontiers in Neurology.

Copyright (c) 2010 Blumberg. This is an open-access article subject to an exclusive license agreement between the authors and the Frontiers Research Foundation, which permits unrestricted use, distribution, and reproduction in any medium, provided the original authors and source are credited. 